\title{
Stellar populations in the bulges of S0s and the formation of SO galaxies
}

\author{
Alfonso Aragón-Salamanca \\ School of Physics and Astronomy, University of Nottingham, Nottingham, NG7 2RD, UK \\ email: alfonso.aragon@nottingham.ac.uk
}

\begin{abstract}
The stellar populations in the bulges of S0s, together with the galaxies' dynamics, masses and globular clusters, contain very interesting clues about their formation. I present here recent evidence suggesting that S0s are the descendants of fading spirals whose star formation ceased.
\end{abstract}

Keywords. galaxies: formation; galaxies: evolution; galaxies: kinematics and dynamics; galaxies: stellar content; galaxies: structure; galaxies: elliptical and lenticular, $\mathrm{cD}$

Lenticular, or S0, galaxies make up some $25 \%$ of large galaxies in the local Universe (Dressler 1980), so understanding how they form must constitute a significant element of any explanation of galaxy evolution. Their location at the crossroads between ellipticals and spirals in Hubble's tuning-fork diagram underlines their importance in attempts to develop a unified understanding of galaxy evolution, but also means that it is not even clear to which of these classes of galaxy they are more closely related.

One often-cited piece of evidence comes from the fact that the proportion of S0s is substantially smaller in distant $(z \sim 0.5)$ clusters than in nearby ones, while spirals show the opposite trend (Dressler et al. 1997), strongly suggesting a transformation from one to the other. However, even if this scenario is accepted, it does not answer the question as to whether S0s are more closely related to spirals or ellipticals, which is intimately connected to the mechanism of transformation. If the transformation simply involves a spiral galaxy losing its gas content through ram pressure stripping (Gunn \& Gott 1972) or "strangulation" (Larson et al. 1980), so ceasing star formation and fading into an S0, then clearly S0s and spirals are closely related. However, it is also possible that mergers can cause such a transformation: while equal-mass mergers between spirals create elliptical galaxies, more minor mergers can heat the original disk of a spiral and trigger a brief burst of star formation, using up the residual gas and leaving an S0. In such a merger scenario, the mechanism for creating an S0 is much more closely related to that for the formation of ellipticals.

Clues to which mechanism is responsible are to be found in the "archaeological record" that can be extracted from spectral observations of nearby S0s. In particular, the presentday stellar dynamics should reflect the system's origins, with the gentle gas stripping of a spiral resulting in stellar dynamics very similar to the progenitor spiral, while the merger process will heat the stars, resulting in kinematics more dominated by random motions, akin to an elliptical. In addition, the absorption line strengths can be interpreted through stellar population synthesis to learn about the metallicity and star formation histories of these systems. Even more interestingly, these dynamical and stellar properties can be compared to see if a consistent picture can be constructed for the formation of each system. I present here some recent evidence suggesting that such a consistent picture is indeed emerging. 


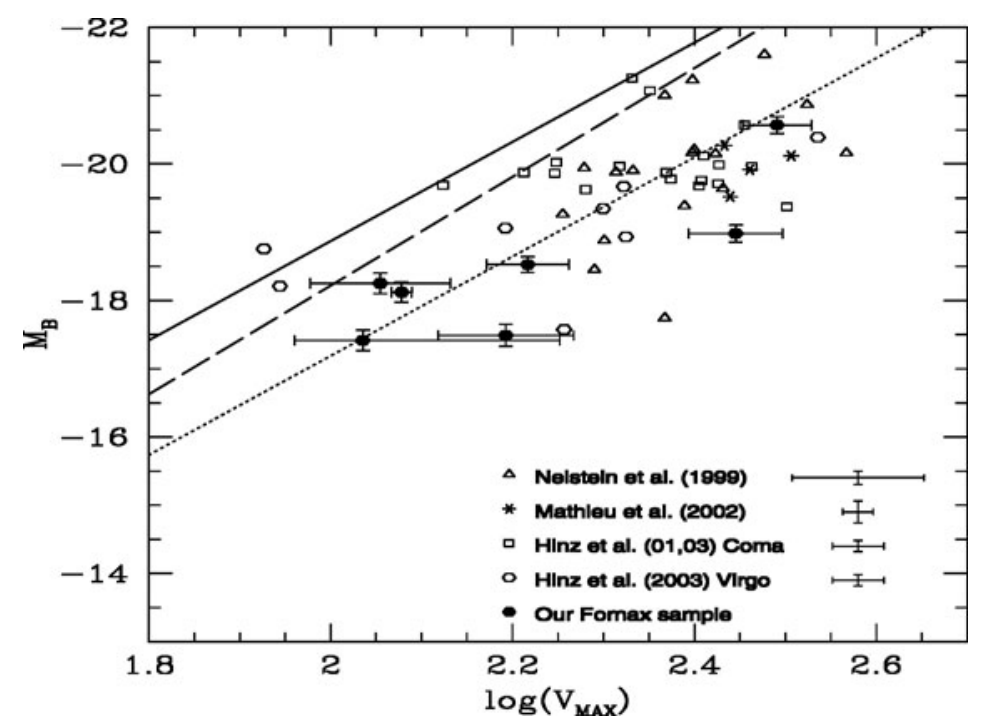

Figure 1. B-band Tully-Fisher relation (TFR) for S0 galaxies using different samples from the literature (open symbols) and our VLT Fornax data (filled circles). The solid and dashed lines show two independent determinations of the TFR relation for local spirals. On average (dotted line), S0s are $\sim 3$ times fainter than spirals at similar rotation velocities (Bedregal, Aragón-Salamanca \& Merrifield 2006b).

\section{Evidence from the Tully-Fisher relation}

Combining published data with high-quality VLT/FORS spectroscopy of sample of Fornax S0s (Bedregal et al. 2006a) we have carried out a combined study of the TullyFisher relation and the stellar populations of these galaxies. Despite the relatively small sample and the considerable technical challenges involved in determining the true rotation velocity $V_{\text {rot }}$ from absorption line spectra of galaxies with significant non-rotational support (see Mathieu et al. 2002), some very interesting results arise. S0s lie systematically below the spiral galaxy Tully-Fisher relation in both the optical and near-infrared (Figure 1). If S0s are the descendants of spiral galaxies, this offset can be naturally interpreted as arising from the luminosity evolution of spiral galaxies that have faded since ceasing star formation. Moreover, the amount of fading implied by the offset of individual S0s from the spiral relation seems to correlate with the luminosity-weighted age of their stellar population, particularly at their centres (Figure 2). This correlation suggests a scenario in which the star formation clock stopped when gas was stripped out from a spiral galaxy and it began to fade into an S0. The stronger correlation at small radii indicates a final last-gasp burst of star formation in this region. See Bedregal, Aragón-Salamanca \& Merrifield (2006b) for details.

\section{Evidence from the globular cluster populations}

Entirely consistent and independent evidence comes from our recent studies of the properties of the globular cluster (GC) systems and stellar populations of SOs (AragńSalamanca, Bedregal \& Merrifield 2006; Barr et al. 2007). If interactions with the intracluster medium are responsible for the transformation of spirals into S0s, the number of globular clusters in these galaxies will not be affected. That is probably not true if more violent mechanisms such as galaxy-galaxy interactions are the culprit (see, e.g., Ashman \& Zepf 1998). If we assume that the number of globular clusters remains constant, the 


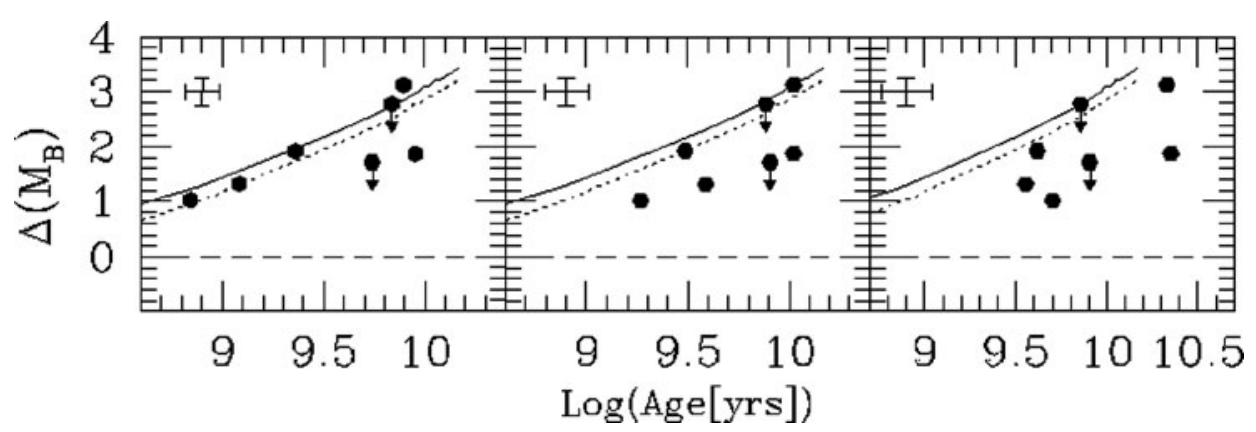

Figure 2. For our VLT Fornax data we plot the shift in magnitudes from the $B$-band spiral TFR versus the stellar population age at the galaxy centre (left panel), at $1 R_{e}$ (middle panel) and at $2 R_{e}$ (right panel). The lines show models for fading spirals. Note that the correlation is strongest for the central stellar populations of the galaxies, suggesting that the last episode of star formation took place there (Bedregal, Aragón-Salamanca \& Merrifield 2006b).

GC specific frequency ( $S_{N} \propto$ number of GCs per unit $V$-band Luminosity) would increase due to the fading of the galaxy. On average, the GC specific frequency is a factor $\sim 3$ larger for S0s than it is for spirals (Aragón-Salamanca et al. 2006), meaning that in the process S0s become, on average, $\sim 3$ times fainter than their parent spiral. Furthermore, in this scenario the amount of fading (or increase in GC specific frequency) should grow with the time elapsed since the star formation ceased, i.e., with the luminosity-weighted age of the S0 stellar population. Figure 3 shows that this is indeed the case, adding considerable weight to the conclusions reached from our Tully-Fisher studies.

\section{Additional evidence from the stellar populations and dynamics}

In Bedregal et al. (2007) we show that the central absorption-line indices in S0 galaxies correlate well with the central velocity dispersions in accordance with what previous studies found for elliptical galaxies. However, when these line indices are converted into stellar population properties, we find that the observed correlations seem to be driven by systematic age and alpha-element abundance variations, and not changes in overall metallicity as is usually assumed for ellipticals. These correlations become even tighter when the maximum circular velocity is used instead of the central velocity dispersion. This improvement in correlations is interesting because the maximum rotation velocity is a better proxy for the S0's dynamical mass than its central velocity dispersion. Finally, the $\alpha$-element over-abundance seems to be correlated with dynamical mass, while the absorption-line-derived ages also correlate with these over-abundances. These correlations imply that the most massive S0s have the shortest star-formation timescales and the oldest stellar populations, suggesting that mass plays a large role in dictating the life histories of SOs.

\section{Conclusions}

The stellar populations, dynamics and globular clusters of S0s provide evidence consistent with these galaxies being the descendants of fading spirals whose star formation ceased. However, caution is needed since significant problems could still exist with this picture (see, e.g., Christlein \& Zabludoff 2004; Boselli \& Gavazzi 2006). Moreover, the number of galaxies studied here is still small, and it would be highly desirable to extend 


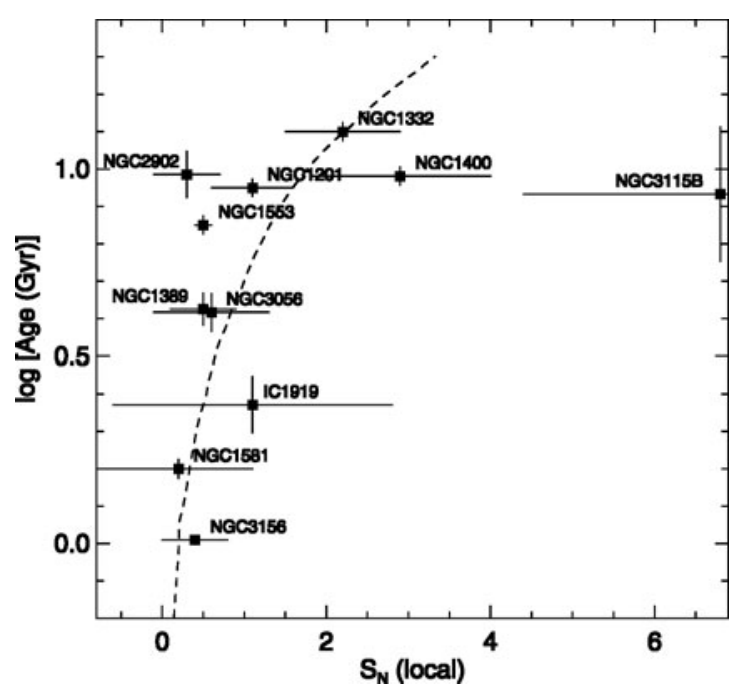

Figure 3. $\log _{10}$ of the luminosity-weighted ages is Gyr vs. the globular cluster specific frequency $\left(S_{N}\right)$ of S0s. The line shows the evolution expected for a fading galaxy according to the stellar population models of Bruzual \& Charlot (2003). The correlation between the fading of the galaxies (or increase in $S_{N}$ ) and the spectroscopically-determined age of their stellar populations is clearly consistent with the predictions of a simple fading model. Note that the $S_{N}$ value for NGC3115B is very unreliable and almost certainly severely overestimated due to contamination from the GC systems of neighbouring galaxies. See Barr et al. (2007) for details.

this kind of studies to much larger samples covering a broad range of galaxy masses and environments.

\section{Acknowledgements}

I thank A.G. Bedregal, M. Merrifield, J.M. Barr, B. Milvang-Jensen, S.P. Bamford and N. Cardiel for allowing me to discuss here results obtained with their help.

\section{References}

Aragón-Salamanca, A., Bedregal, A. G., \& Merrifield, M. R., 2006, A\&\&A, 458, 101

Ashman, K. M. \& Zepf, S. E. 1998, Globular cluster systems (Cambridge, U.K.; New York: Cambridge University Press, Cambridge astrophysics series; 30)

Barr, J. M., Bedregal, A. G., Aragón-Salamanca, A., Merrifield, M. R., \& Bamford, S. P. 2007, $A \mathscr{E} A, 470,173$

Bedregal, A. G., Aragón-Salamanca, A., Merrifield, M. R., \& Milvang-Jensen, B. 2006a, MNRAS, 371,1912

Bedregal, A. G., Aragón-Salamanca, A., \& Merrifield, M. R. 2006b, MNRAS, 373, 1125

Bedregal, A. G., Aragón-Salamanca, A., Merrifield, M. R., \& Cardiel, N. 2007, MNRAS, submitted

Boselli, A. \& Gavazzi, G. 2006, MNRAS, 118, 517

Bruzual, G. \& Charlot, S. 2003, MNRAS, 344, 1000

Christlein, D. \& Zabludoff, A. I. 2004, ApJ, 616, 192

Dressler, A. 1980, ApJ, 236, 351

Dressler, A., et al. 1997, ApJ, 490, 577

Gunn, J. E. \& Gott, J. R. I. 1972, ApJ, 176, 1

Larson, R. B., Tinsley, B. M., \& Caldwell, C. N. 1980, ApJ, 237, 692

Mathieu, A., Merrifield, M. R., \& Kuijken, K. 2002, MNRAS, 330, 251 